\title{
Experimental Evaluation For Joint Estimation Approach ${ }^{1}$
}

\author{
Y. Zhang ${ }^{2}$, J.A. Fessler ${ }^{3}$, N.H. Clinthorne ${ }^{4}$ and W.L. Rogers ${ }^{4}$ \\ ${ }^{2}$ Department of Radiology, ${ }^{3}$ Department of EECS and ${ }^{4}$ Division of Nuclear Medicine, The University of Michigan, Ann Arbor.
}

\begin{abstract}
Single Photon Emission Computed Tomography (SPECT) provides a potential to perform in vivo quantification of the radioactivity and dose distributions in the process of evaluating radiopharmaceuticals. The inherent modest resolution in SPECT impedes the potential of accurate quantification. Previously, we investigated a joint estimation approach for combining SPECT functional information with high resolution, structurally correlated MRI anatomical information to improve the accuracy of SPECT quantification, and the computer simulation results showed that this approach can exploit MRI region information that matches the SPECT functional information and to reduce artifacts caused by mismatched MRI anatomical information. In this paper, we further describe the experimental evaluation of the joint estimation approach using actual SPECT and MRI imaging with an animal-sized phantom. We will describe practical details in applying the joint estimation approach and present the experimental evaluation results of quantitative analysis.
\end{abstract}

\section{INTRODUCTION}

All radiopharmaceuticals used in nuclear medicine have to be evaluated for their target specificity measured by the tissue radiotracer uptake distribution as a function of time in small laboratory animals. Conventional in vitro evaluation procedure by dissecting and counting has some disadvantages since the animal must be sacrificed to determine tracer activity or concentration. Thus to obtain the temporal response, multiple animals must be sacrificed at each of many time points. Measurement variation is thus increased due to differences among animals. It would be more desirable to measure the consecutive response from the same animal. In vivo estimation of radiotracer uptake distribution based on a reconstruction of 3D emission tomographic images as a function of time can overcome the aforementioned drawbacks, since the same animal is imaged repeatedly over the entire time course. Single Photon Emission Computed Tomography (SPECT) has the potential to perform this type of in vivo estimation, provided acquisition, reconstruction and compensation procedures are adequately implemented and utilized[2]. However, the spatial resolution of reconstructed SPECT images is inherently limited due to statistical fluctuations in photon distribution, tradeoffs between detection efficiency and collimator resolution, limited

\footnotetext{
${ }^{1}$ This research was supported in part by the National Cancer Institute under grant No. CA-54362 and Rackham Predoctoral Fellowship. The University of Michigan. The authors want to thank to Drs. Swanson and Wrobel for the help in experiments.
}

photons statistics due to dose restriction, and Compton scatter effect. Limited spatial resolution causes erroneous estimates of uptake dose distribution and total uptake within an organ. On the other hand, MRI images have better spatial resolution and anatomical delineation than SPECT images. Since the normal function of most organs is often correlated with their anatomical structures[3], it is therefore natural for us to expect that applying high resolution, structurally correlated MRI region side information has the potential to reduce the SPECT quantification error in determining the total activity or average concentration of tracer in a given organ or ROI. However, in many practical situations the MRI anatomical region may not be identical to the SPECT functional region because the signals of the two imaging systems are derived from entirely different physical processes. Simulation results show that using side information directly is unlikely to be significantly beneficial for the quantification task if there is mismatch[4]. Some effort has been made to correct the effect of the mismatches [4]. We have also investigated a joint estimation approach to reduce the effect caused by the mismatch while keeping the benefit of high resolution MRI anatomical information[1]. A joint Penalized Maximum Likelihood (JPML) objective function, is used with an joint Space-Alternating Generalized Expectation-Maximization algorithm (JSAGE) derived upon on SAGE algorithm[5] for SPECT reconstruction. The objective function incorporates statistical distributions of both SPECT acquisition and MRI region measurement, so that the SPECT pixel intensity and functional region are jointly estimated from both SPECT and MRI data. In [1], our computer simulation results showed that the joint estimation approach with JSAGE algorithm surmounts the difficulty of the mismatch between SPECT functional region and MRI anatomical region, and improved the accuracy of the SPECT quantification.

Beyond computer simulations, we have performed actual SPECT acquisition of an animal-sized phantom with an Animal Single Photon Ring Tomograph (ASPRINT) developed in our laboratory by Rogers, etal[6] and actual MRI acquisition of the same phantom with GE CSI2T unit to evaluate the joint estimation approach. In this paper, we will describe the theoretical derivation of the joint estimation approach (Section II), and some practical issues of applying the approach, such as modeling the photon acquisition process, registration and segmentation of registered MRI images (Section III). We will present the experimental evaluation result of the joint estimation approach in terms of image visual quality and radioactivity quantification (Section IV). Section $V$ discusses problems associated with the joint estimation and possible solutions as future work. 


\section{JOINT ESTIMATION APPROACH}

In the joint estimation approach, we choose region labels to convey the anatomical regions extracted from MRI, because region labels more easily encourage region contiguity, and region labels need half as many the parameters as line sites. Furthermore, it is also relatively easier to handle region labels when extending the method to 3D cases. Thus each SPECT pixel(voxel) $\lambda_{k}$ has a corresponding region label site, $l_{k}$. However, the region labels $l$ are not simply a "copy" from the MRI region information. They are treated as random variables just as the SPECT mean intensity variables. After being initialized with the segmented MRI, they are jointly estimated with SPECT pixel mean intensity variables $\lambda$, using a JPML objective. We update a SPECT mean intensity pixel and its corresponding region label simultaneously, such that a label-pixel pair updating is mutually constrained. Since region labels are estimated jointly from both segmented MRI and SPECT data, only those parts of the MR anatomical regions that match the SPECT functional regions are represented by the estimated labels, and constrain the SPECT intensity reconstruction. The mismatched region labels will be updated during the joint estimation process, to approach the SPECT functional region. Thus the artifacts due to mismatch are reduced.

\section{A. Objective Function}

The JPML objective is as the following:

$$
\boldsymbol{\Phi}_{\text {joint }}(\boldsymbol{\lambda}, \boldsymbol{l})=L(\boldsymbol{y}, \tilde{\boldsymbol{l}} \mid \boldsymbol{\lambda}, \boldsymbol{l})-U(\boldsymbol{\lambda}, \boldsymbol{l}),
$$

where $y$ is the SPECT projection data, $\tilde{l}$ is the MR anatomic region measurement, $\boldsymbol{\lambda}$ and $\boldsymbol{l}$ are SPECT intensity and label parameters to be estimated, $L$ is the joint log-likelihood incorporating both the SPECT noise distribution and the MRI side information measurement statistics, and $U$ is a Gibbs type joint penalty terms. Since SPECT and MRI are independent imaging processes, their joint log likelihood function conditioned on $\lambda$ and $l$ is separable, thus the log-likelihood: $L(\boldsymbol{y}, \tilde{\boldsymbol{l}} \mid \boldsymbol{\lambda}, \boldsymbol{l})=L(\boldsymbol{y} \mid \boldsymbol{\lambda})+L(\boldsymbol{l} \mid \boldsymbol{l})$. The SPECT measurements have Poisson distributions, thus :

$$
L(\boldsymbol{\lambda}, \boldsymbol{y})=\log f(\boldsymbol{y} \mid \boldsymbol{\lambda}) \equiv \sum_{n}\left(-\bar{y}_{n}(\boldsymbol{\lambda})+y_{n} \log \bar{y}_{n}(\boldsymbol{\lambda})\right),
$$

where $\bar{y}_{n}(\boldsymbol{\lambda})=\sum_{k} a_{n k} \lambda_{k}+r_{n}$ and $a_{n k}$ are the elements of system matrix, $r_{n}$ is the acquisition background radiation term, " $\equiv$ " represents the equivalence due to a term independent of $\lambda$. We assume that the MRI has additive electrical noise, which can be modeled as white Gaussian noise[7], and different regions have different intensities. If MRI is segmented using simple pixel-by-pixel thresholds, then the log-likelihood for $\tilde{l}_{k}$ given $l_{k}$ is: $L(\tilde{l} \mid l)=\sum_{k=1}^{p} \log P\left(\tilde{l}_{k} \mid l_{k}\right)$, where $P\left(\tilde{l}_{k} \mid l_{k}\right)$ is the probability of assigning the label $\tilde{l}_{k}$ to the $k$ th pixel during segmentation given $l_{k}$ is the true label. The $P\left(l_{k} \mid l_{k}\right)$ also incorporates the possibility of existing mismatch in the segmented MRI.

The joint penalty term $U(\lambda, l)$ can be viewed as a log-prior pdf of the SPECT pixels and region labels. We adopt a hierarchical form of $U$, thus:

$$
U(\lambda, l)=\frac{1}{\beta_{1}} U_{1}(\lambda \mid l)+\frac{1}{\beta_{2}} U_{2}(l),
$$

where $U_{1}(\boldsymbol{\lambda} \mid \mathbf{l})$ is the log-prior pdf of $\boldsymbol{\lambda}$ conditioned on region labels $l, U_{2}(l)$ is the log-prior pdf of region labels, $\beta_{1}$ and $\beta_{2}$ are the regularization parameters. The prior knowledge of the joint pixel-label lattice sites is that the pixels tend to have similar intensity if the underlying labels are the same, and the labels tend to be the same if the corresponding pixel intensities are similar. The prior knowledge for regions is that they are contiguous, regularly shaped and have small number of regions. To encourage smoothness within the same region but allow discontinuities between regions in the SPECT image, we apply weighted Gibbs function[4] to $U_{1}$ :

$$
U_{1}(\boldsymbol{\lambda}, \boldsymbol{l})=\frac{1}{2} \sum_{k=1}^{p} \sum_{j \in \mathcal{N}_{k}} \omega_{k j}(l) \psi\left(\lambda_{k}-\lambda_{j}\right),
$$

where $\psi\left(\lambda_{k}-\lambda_{j}\right)$ is usually a quadratic term, and $\mathcal{N}_{k}$ is the neighborhood of pixel $k$. Usually a 2nd-order neighborhood is used. Then the weights $\omega_{k j}$ link the pixel $k$ with its neighbors $j \in \mathcal{N}_{k}$, and can be defined as:

$$
\omega_{k j}(l)= \begin{cases}1 \text { (direct neighbor) } & \text { if } l_{k}=l_{j} \\ \sqrt{2} / 2 \text { (diagonal neighbor) } & \text { if } l_{k}=l_{j} \\ 0 & \text { if } l_{k} \neq l_{j}\end{cases}
$$

When $\psi\left(\lambda_{k}-\lambda_{j}\right)$ is large, the minimization of the penalty pushes the current label $l_{k}$ to be different from its neighbors. This penalty term associates the SPECT intensity vector $\lambda$ with the label vector $l$, so their estimates are mutually constrained. This avoids the domination of mismatched labels extracted from MRI over the SPECT reconstruction. To favor region contiguity prior, we choose $U_{2}$ as:

$$
U_{2}(l)=\sum_{k=1}^{p} \sum_{j \in \mathcal{N}_{k}} \omega_{k j}(l),
$$

where $\omega_{k j}$ is the same as defined in equation (5).

\section{B. Reconstruction Algorithm}

We apply an iterative SAGE algorithm[5], with slight modification for our JPML objective, because it converges fast and ensures a monotonic increase in objective function. To maximize the function $\Phi_{j o i n t}(\lambda, l)$ with SAGE, the conditional expectation of the likelihood the SAGE algorithm can be straightforwardly modified to jointly maximize the function $\phi_{k}\left(\lambda_{k}, l_{k} ; \lambda^{i}, l^{i}\right)$, with respect to each pixel-label pair, $\left(\lambda_{k}, l_{k}\right)$, by holding the remaining current pixel-label pairs $\left(\lambda_{-k}^{i}, l_{-k}^{i}\right)$ fixed as:

$$
\begin{aligned}
\left(\lambda_{k}^{i+1}, l_{k}^{i+1}\right) & =\arg \max _{\left(\lambda_{k}, l_{k}\right)} \phi_{k}\left(\lambda_{k}, l_{k} ; \lambda^{i}, l^{i}\right) ; \\
\phi_{k}\left(\lambda_{k}, l_{k} ; \lambda^{i}, l^{i}\right) & =Q_{\mathbf{X}_{k}^{i}}\left(\lambda_{k}, \lambda^{i}\right)+L\left(l_{k} ; \tilde{l}_{k}\right)- \\
\frac{1}{\beta_{1}} U_{1}\left(\lambda_{k}, \lambda_{-k}^{i} ; l_{k}, l_{-k}^{i}\right) & -\frac{1}{\beta_{2}} U_{2}\left(l_{k}, l_{-k}^{i}\right) ;
\end{aligned}
$$


where $Q_{\mathbf{X}_{k}^{i}}\left(\lambda_{k}, \lambda^{i}\right)$ is the conditional expectation of log-likelihood defined on the hidden-data space $\mathbf{X}_{k}^{i}$, $L\left(l_{k} ; \tilde{l}_{k}\right)=\ln P\left(\tilde{l}_{k} \mid l_{k}\right), i$ is the iteration index, and $U_{1}(\cdot)$ and $U_{2}(\cdot)$ are re-defined as:

$$
\begin{aligned}
U_{1}\left(\lambda_{k}, \lambda_{-k}^{i} ; l_{k}, l_{-k}^{i}\right) & =\frac{1}{2} \sum_{j \in \mathcal{N}_{k}} \omega_{k j}(l)\left(\lambda_{k}-\lambda_{j}\right)^{2} \\
U_{2}\left(l_{k}, l_{-k}^{i}\right) & =\sum_{j \in \mathcal{N}_{k}} \omega_{k j}(l) .
\end{aligned}
$$

The joint update procedure for each $\left(\lambda_{k}, l_{k}\right)$ pair is done in two steps because of the discrete feature of the label, $l$ :

1. For each possible state of $l_{k}$, say $l_{k}^{*}$, fix $l_{-k}^{i}$ and $\lambda_{-k}^{i}$, seek $\lambda_{k}^{*}$ such that: $\lambda_{k}^{*}=0$; or $\left.\frac{\partial}{\partial \lambda_{k}} \phi_{k}\left(\lambda_{k}, l_{k}^{*} ; \lambda^{i}, l^{i}\right)\right|_{\lambda_{k}=\lambda_{k}^{*}}=$ 0 , if $\lambda_{k}^{*}>0$.

2. Evaluate $\phi_{k}\left(\lambda_{k}^{*}, l_{k}^{*} ; \lambda^{i}, l^{i}\right)$ for each of the possible pairs $\left(\lambda_{k}^{*}, l_{k}^{*}\right)$, choose the one that maximizes $\phi_{k}$, and define it as the $\left(\lambda_{k}^{i+1}, l_{k}^{i+1}\right)$.

The new objective, $\phi_{k}\left(\lambda_{k}, l_{k} ; \lambda^{i}, l^{i}\right)$ contains mixed discrete and continuous variables. The conventional definition of convexity does not apply to this objective function, and the global convergence is not guaranteed in this iterative reconstruction. We thus used a deterministic annealing procedure[8] by applying a sequence of penalty functions with an exponentially varying $\beta_{2}$.

\section{PRACTICAL IsSUES OF THE APPROACH}

\section{A. Statistical Model of ASPRINT acquisition}

In a general SPECT system, the projection acquisition can be modeled as:

$$
\boldsymbol{Y} \sim \operatorname{Poisson}(\boldsymbol{A} \cdot \boldsymbol{\lambda}+\boldsymbol{r}),
$$

where $Y$ is the measured projection vector in a photo-peak energy window, $\lambda$ is the pixel vector, $\boldsymbol{A}$ represents the system response weights matrix, and $r$ represents the mean contribution to the projection from background radiation. In ASPRINT, the system weights matrix is calculated according to the system geometry and experimentally obtained detector mean response. ASPRINT provides the azimuthal collimation with a slit aperture ring. Both edges of each slit have depleted uranium (DU) to reduce the penetration of photons through the edge area. However, the DU emits small amount of $\gamma$ photons in a wide energy range. This affects the photo-peak projection acquisition. And we have observed that the projection of DU looks like white noise without any spatial dependency to the slit position, and the energy spectrum of slit edge radiation looks flat in the energy around the photo-peak window [9]. In addition to DU, Compton scatter effect also contributes to the photo-peak projection. And the projection data from scattered photons are very smooth [10]. We propose a statistical model for the projection in the photo-peak window to include the background radiation and Compton scatter effect:

$$
\boldsymbol{Y}_{p} \sim \operatorname{Poisson}\left\{\boldsymbol{P}_{e} \cdot\left(\boldsymbol{A} \times \boldsymbol{\lambda}+\overline{\boldsymbol{Y}}_{s}+\overline{\boldsymbol{Y}}_{u}\right)\right\},
$$

where $\boldsymbol{Y}_{p}$ is the photo-peak projection, $\overline{\boldsymbol{Y}}_{s}$ and $\overline{\boldsymbol{Y}}_{u}$ are the mean of the scattered photons and the mean of the room background plus DU photons contributing to the photo-peak projection, $\boldsymbol{P}_{e}$ is the measured detector element efficiency, and $\boldsymbol{A}$ is the system weights matrix, which may include attenuation factors. Here the $\overline{\boldsymbol{Y}}_{s}$ and $\overline{\boldsymbol{Y}}_{u}$ can be regarded as a background radiation term, $\boldsymbol{r}$, in equation (9). To estimate $\overline{\boldsymbol{Y}}_{s}$ and $\overline{\boldsymbol{Y}}_{u}$, we apply a simultaneous three-iso-width-energy-window acquisition method. They are scatter, photo-peak, and background windows from low to high energy. We use the projection acquired in the background window to estimate the mean contribution from depleted uranium in the photo-peak window, and use the partial of the projection acquired in the scatter window to estimate the mean contribution from scattered photons, as:

$$
\begin{aligned}
& \hat{\overline{\boldsymbol{Y}}}_{u}=\operatorname{smooth}\left(\boldsymbol{P}_{e}^{-1} \cdot \boldsymbol{Y}_{b w}\right) \\
& \hat{\boldsymbol{Y}}_{s}=K\left(\overline{\boldsymbol{Y}}_{s w}-\hat{\overline{\boldsymbol{Y}}}_{u}\right)
\end{aligned}
$$

where $\overrightarrow{\boldsymbol{Y}}_{u}$ is the mean of background window projection, $\overline{\boldsymbol{Y}}_{s w}$ is the mean of the scatter window projection, which can be estimated by $\operatorname{smooth}\left(\boldsymbol{P}_{e}^{-1} \cdot \boldsymbol{Y}_{s w}\right), \boldsymbol{Y}_{b w}$ and $\boldsymbol{Y}_{s w}$ are the projection acquisition in scatter and background windows respectively. $\quad \boldsymbol{P}_{e}^{-1}$ is detector efficiency correction, and $K$ is the scatter multiplier estimated from simple dual-window correction method [11]. Thus the model is actually:

$$
\boldsymbol{Y}_{p} \sim \text { Poisson }\left\{\boldsymbol{P}_{e} \cdot\left(\boldsymbol{A} \boldsymbol{\lambda}+K\left(\overline{\boldsymbol{Y}}_{s w}-\overline{\boldsymbol{Y}}_{b w}\right)+\overline{\boldsymbol{Y}}_{b w}\right)\right\} .
$$

The JSAGE is thus based on the above model.

\section{B. Phantom Design}

We made an animal sized phantom composed of a phantom body with three different syringes aligned parallelly in axial direction, and a leak-proof lid, see Fig.1. The syringes are filled with mixtures of $\mathrm{TcO}_{4}$ and Gd-DTPA (MRI contrast agent) with different concentrations. There are two sets of ten fiducial markers on body surface, one axial and one helical. To simulate a missing region, the solutions in the center syringe and inside phantom body do not have Gd-DTPA.

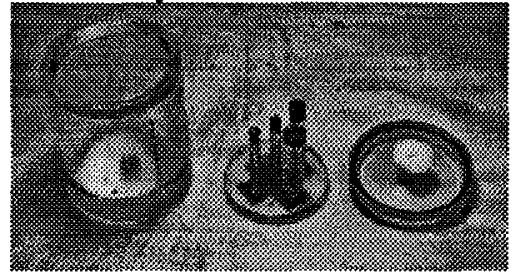

Fig. 1 A photograph of 2D phantom.

\section{Registration and Segmentation}

To be able to apply MRI region information, a registration is performed between SPECT and MRI using the estimated coordinates of fiducial markers in the SPECT filteredbackprojection(FBP) reconstruction and acquired MRI slices. Levenberg-Marquardt algorithm is applied to obtain the LS estimation of rigid body transformation parameters. The MRI 
images are then transformed into SPECT image domain using the estimated parameters and tri-linear interpolation.

Because of Gd-DTPA, all the regions in MRI are well differentiated from its background region. We estimate the mean and variance of each region. Under the Gaussian assumption, we apply Maximum $A$ Posteriori (MAP) criterion and likelihood ratio test to seek the threshold to segment regions and calculate the probability $P(\tilde{l} \mid l)$. However, if we suspect that there might be a new functional region $D$ in SPECT while there is no such region MRI by observing corresponding FBP and MRI slice, as shown in Fig.2, then we will assign a probability $P(\tilde{l}=C \mid l=D)$. Usually it is close to 1 .



Fig. 2 Compare FBP and MRI for $P\left(\tilde{l}_{k} \mid l_{k}\right)$ where probable mismatch exists.

\section{EXPERIMENTAL EVALUATION RESULTS}

We performed similar evaluations as we did in the computer simulations[1]. Fig. 3 shows the reconstructed images of the center slice of the phantom using different methods. (a) is from FBP, we see that the image is noisy and boundaries are blurred; (b) is from PML-SAGE, without any region information, the smoothness is achieved at the cost of global blurring; (c) is from PML-SAGE with weighted Gibbs penalty where the weights are defined by the MRI region. We see that for those regions whose corresponding MRI regions are available, the region are smooth and boundaries are sharp. However, for the center region whose corresponding MRI region is missing, after the reconstruction, we see the blurring effect; (d) is from joint estimation where the region labels are initialized with the MRI region. We see that the center region is recovered during the joint estimation and the other regions remain good with MRI region information. Fig.4 and Fig.5 show 15 slices of

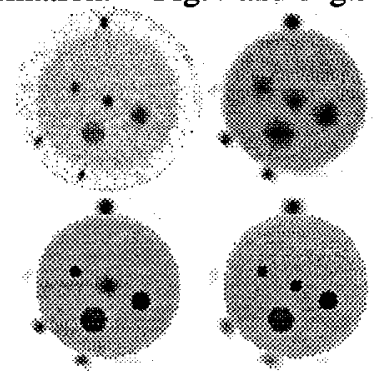

Fig. 3 Reconstruction of the center slice (slice 9) from different methods. (a): Top left; (b): Top right (c): Bottom left (d): Bottom right

image intensities and region labels from the joint estimation

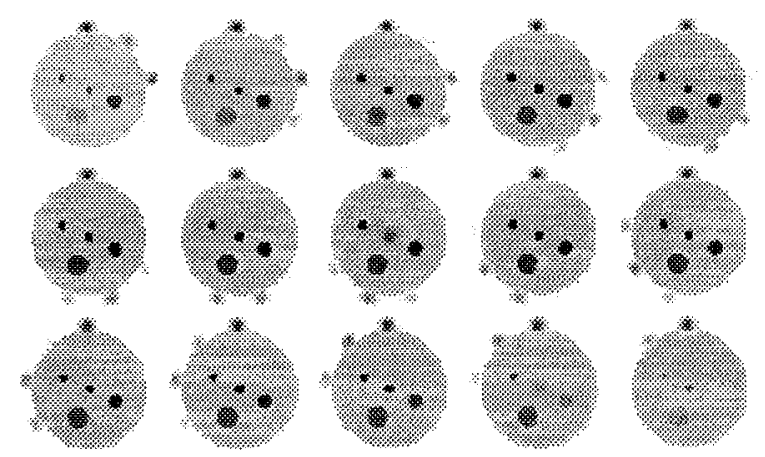

Fig. 415 consecutive image slices of the phantom from joint estimation approach. The spots around are fiducial markers.

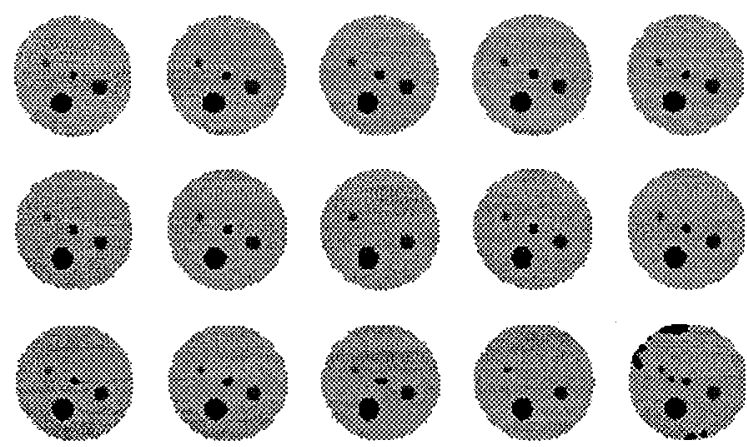

Fig. 515 slices of region labels from joint estimation approach.

approach. Because of the non-convexity of the objective function, global convergence is not guaranteed even though deterministic annealing is applied. The problem is illustrated in slice 8 and slice 14 . The center hot region labels are not recovered by joint estimation, and consequently, the intensity image has a blurred boundary for the center region.

We also applied bias vs. STD curve evaluation to obtain an objective comparison among the reconstruction approaches in terms of the estimated total activities in different objects (syringes) using 30 ASPRINT consecutive acquisition of the phantom.

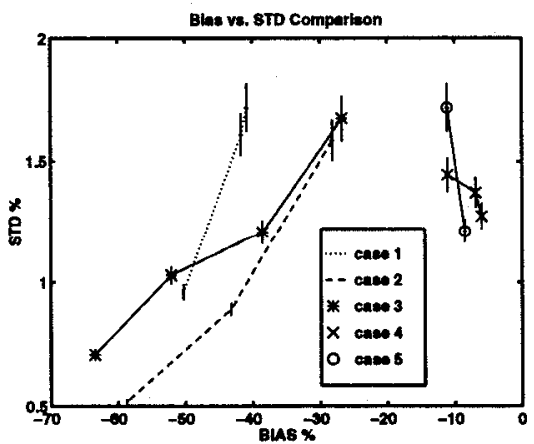

Fig. 6 Bias vs. STD comparison for the estimated total activity in the small syringe near the edge of the phantom (no mismatch). The error bars of the estimations of both bias and STD are shown for each point.

Fig.6 shows the bias vs. STD curves for the total activity estimated for the small hot syringe near the edge of the 
phantom. Case 1 is from fan-beam FBP with 3rd-order Butterworth windows of different cut-off frequencies. Case 2 is from fan-beam FBP with Wiener filters with different cut-off frequencies. Case 3 is from PML-SAGE with spatially-invariant weighted Gibbs penalty. Case 4 is from PML-SAGE with weighted Gibbs penalty where the weights are defined by MRI region information, and Case 5 is from joint estimation where the region label variables are initialized with the same region. Apparently, Case 4 and 5 perform better than Case 1, 2, and 3, because the correct MRI region information improves the quantitative evaluation of the total activity of the region of interest as we have shown in simulations. In addition, the closeness of Case 4 and Case 5 shows that when the MRI region information matches the SPECT region information, the performance of joint estimation is similar to the "direct" application of MRI side information in PML-SAGE(Case 4).

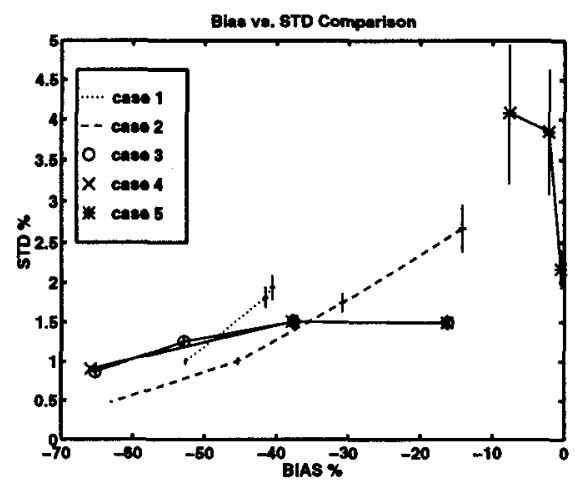

Fig. 7 Bias vs. STD comparison for the estimated total activity in the small syringe at the center of the phantom. Error bars of the estimations of both bias and STD are shown for each point.

Fig.7 shows the bias vs. STD curves for the total activity estimated for the small hot syringe at the center of the phantom. Case 1, 2,3,4 and 5 are the same as for Fig.6. In this situation, MRI region information for the center hot region is missing, so that the performance of "direct" application of MRI information in PML-SAGE with weighted Gibbs penalty (Case 4) comes close to the performance of PML-SAGE with spatially-invariant weighted Gibbs penalty(Case 3), and also close to the FBP reconstruction. This fact confirms that when using missing region information "directly" to define the weights, the performance is no better than those without region information. In the joint estimation(Case 5), because of the recovery of the center region, the curve illustrates reduced estimation bias. Here the unrecovered region cases in joint estimation were excluded in bias-variance calculation.

\section{SUMMARY AND DISCUSSION}

We have proposed a joint estimation approach which incorporates MRI anatomical region information into SPECT reconstruction to improve SPECT functional tracer distribution quantification based on a joint PML with JSAGE algorithm. It exploits the MRI region information that matches the SPECT functional information and to reduce the artifacts caused by mismatch between MRI anatomical region information and
SPECT functional region information. The evaluations of bias vs. STD trade-offs of experimental results with matched and missing MRI region information show that the aim has been accomplished. However, the global convergence of the joint estimation approach is not guaranteed due to the non-convex property of the joint PML objective function, caused by the discrete nature of the label variables in the objective function. One possible solution is to apply a continuous label model. Originally, a region label variable was designed as a scalar variable, $l$, which takes one value out of an integer set with each integer representing a region type. In the new model, each label $L$ is a vector variable, $l$, with each element representing the probability of the voxel site belonging to a region, and the elements' sum is equal to 1 . Here each element is a continuous variable. This is probable to improve convergence property for the JPML objective function.

\section{REFERENCES}

[1] Y. Zhang, J.A. Fessler, N.H. Clinthorne, W.L. Rogers, "Incorporating MRI Region Information Into SPECT reconstruction Using Joint Estimation", Proceedings of IEEE 1995 International Conference on Acoustic, Speech and Signal Processing, Vol. 4, pp. 2307-2310, 1995.

[2] R.J. Jaszczak, K.L. Greer, and R.E. Coleman, "SPECT Quantification of Regional Radionuclide Distribution", Fourth International Radiopharmaceutical Dosimetry Symposium, Nov. 5-8, pp. 82-96, 1985, Oak Ridge, Tennessee, Oak Ridge Associated University.

[3] G. Gindi, M. Lee, A. Rangarajan, and I. Zubal, "Bayesian Reconstruction of Functional Images Using Anatomical Information as Priors", IEEE Trans. on Medical Imaging, Vol. 12, No. 4, pp. 670-680, Dec. 1993

[4] J. Fessler, N. Clinthorne, L. Rogers, "Regularized Emission Image Reconstruction Using Imperfect Side Information", IEEE Trans. on Nuclear Science, Vol. 39, No. 5, pp. 1464-1471, Oct. 1992.

[5] J.A. Fessler and A.O. Hero, "Space-Alternating Generalized EM Algorithms for Penalized Maximum-Likelihood Image Reconstruction", Comm. and Sign. Proc. Lab., Dept. of EECS, Univ. of Michigan, Ann Arbor, MI, Tech. Rep. 286, Feb. 1994.

[6] W.L. Rogers, N. Clinthorne, X. Liu, L. Shao, R. Wahl, "Design of ASP: A High-Resolution Tomograph for Small Animals", J. Nucl. Med. ; 31(5): 769, 1990.

[7] W.A. Edelstein, G.H. Clover, C.J. Hardy, and R.W. Redington, "The Intrinsic Signal to Noise Ratio in NMR Imaging", Magnetic Resonance in Medicine, Vol. 3, pp. 606-618, 1986.

[8] S. Geman and D. Geman, "Stochastic Relaxation, Gibbs Distributions and the Bayesian Restoration of Images", IEEE Trans. on Pattern Analysis and Machine Intelligence, Vol PAMI-6 No.6 pp. 721-741, Nov. 1984.

[9] Yong Zhang, "Improved SPECT Radioactivity Quantification Using MRI Side Information", Ph.D. thesis, Department of Biomedical Engineering, The University of Michigan, Ann Arbor, 1996.

[10] R.J. Jaszczak, K.L. Greer, C.E. Floyd, Jr., C. Harris, and R.E. Coleman, "Improved SPECT Quantification Using Compensation for Scattered Photons", Journal of Nuclear Medicine, Vol. 25, No. 8, pp. 893-900, 1984

[11] R.J. Jaszczak, C.E. Floyd, R.E. Coleman, "Scatter compensation techniques for SPECT”, IEEE Trans. on Nuclear Science, NS-32, pp. 786-793, 1985. 